\title{
Evaluation of a T2DM Lifestyle Modification Counseling Program to Improve Patient Engagement in Diabetes Self-Care in a Community Mental Health Clinic
}

\section{Grace Adeola Adepoju}

New Beginning Community Mental Health Center, 2780 3rd Ave, The Bronx, NY 10455, USA

\section{Abstract}

Introduction: The prevalence of Type 2 diabetes mellitus (T2DM) is gradually increasing throughout the globe. An estimated 415 million adults worldwide have diabetes. It is estimated that another 318 million adults suffer from impaired glucose tolerance. By the year 2040, these numbers are expected to increase to 642 million and 482 million respectively. The disease is reaching pandemic proportions and represents a significant burden on the world population. The use of lifestyle modification counseling has been shown to statistical significant among patient with T2DM, however, diabetes still a major problem in develop and under develop country. The project aimed to increase patient engagement in care of T2DM in one community mental health center (CMHC). Participants included a convenience sample of mental health patients attending the CMHC. The theoretical framework for the project was the trans-theoretical model of quality improvement.

Methods: This project utilized a descriptive survey design. A total of 73 patients participated in both the preand post-interventions. The average age was $59.36(\mathrm{SD}=8.739)$ years old with the youngest participant being 40 years old and the most senior participant being 78 years old for an age range of 38 years. As seen in Table 1 , the participants were mostly female $(\mathrm{n}=52,71.2 \%)$, Hispanic $(\mathrm{n}=59,80.8 \%)$ and were diagnosed with depression $(n=39,53.4 \%)$.Participating patients completed the Patient Activation Measure 13 (PAM13) at pre- and post- intervention intervals to assess individual attitudes and confidence related to the selfmanagement of their care. Analysis included chart audits to assess the effectiveness of the intervention and to address the feasibility of implementing a lifestyle modification counseling program.

Results: Patients demonstrated significantly increased engagement in their care at the end of the twelve-week implementation period. The PAM13 survey results remained consistent, and patients felt confident in their ability to effectively continue engagement in their care. Patient engagement in T2DM care improved after lifestyle modification counseling. The potential to improve health care outcomes is evident when providers increase communication with patients. Such interventions may ultimately lead to lower overall health care costs.

Conclusions: The results demonstrated that an increase in post-implementation lifestyle modification counseling helps patient engagement. The intervention was statistically significant and demonstrated that the introduction of a lifestyle modification counseling increased patient engagement.

\section{Introduction}

Type 2 diabetes mellitus (T2DM) is a metabolic disorder, the prevalence of which continues to dramatically increase around the globe. The International Diabetes Federation [1] estimated that 425 million adults worldwide have developed diabetes. By the year 2045, this number is expected to increase to 629 million adults with diabetes and 482 million people with impaired glucose tolerance [1] The disease is reaching pandemic proportions and represents a significant burden on countries worldwide, especially poorly developed countries [2].

People living with T2DM are more vulnerable to various forms of both short- and long-term complications, which frequently place them at risk for premature death. Additionally, the IDF [1] reported that the number of people with T2DM will continue to increase by $80 \%$ in low-income and middle-income countries. The incidence of T2DM is varied across geographical regions due to two factors: environment and lifestyle [1]. Many interventions, such as lifestyle modification and pharmacological treatment, have been evaluated to demonstrate that T2DM can be prevented or delayed in high-risk individuals with impaired glucose tolerance [3]. The spread and intensity of T2DM must be controlled to reduce the burden of chronic illness on society.
To that end, caring for patients in a community mental health program with co-occurring diabetes demands more than knowledge of clinical practice guidelines. Close examination of the literature revealed diabetes burdens and complications that are unique to Hispanic and Black Americans with mental health problems in the United States [3]. In a study conducted by the Centers for Disease Control and Prevention [4], in which the researchers examined diabetes among Hispanic and Black American individuals, poor dietary habits, physical inactivity, distress and depression associated with diabetes, and poor diabetes management were endemic. Yoon, Kwok, and Magkidis's [5] systematic literature review revealed that T2DM can be easily prevented through lifestyle modification, dietary control, and weight loss. Therefore, educating

"Corresponding Author: Dr. Grace Adeola Adepoju, New Beginning Community Mental Health Center, 2780 3rd Ave, The Bronx, NY 10455, USA; E-mail: gadepoju2016@gmail.com

Citation: Adepoju GA (2020) Evaluation of a T2DM Lifestyle Modification Counseling Program to Improve Patient Engagement in Diabetes Self-Care in Community Mental Health Clinic. Int J Nurs Clin Pract 7: 319. doi: https://doi. org/10.15344/2394-4978/2020/319

Copyright: (C) 2019 Adepoju. This is an open-access article distributed under the terms of the Creative Commons Attribution License, which permits unrestricted use, distribution, and reproduction in any medium, provided the original author and source are credited. 
the public remains a vital component to ameliorating the effects of this devastating disease. Despite new insight into the pathophysiology of the disease, and the wide range of drugs that have been developed to treat the disease, a cure remains out of reach for the foreseeable future [5]. However, individually tailored self-management protocols will improve patient quality of life and the treatment outcomes for T2DM [6].

Diabetes is a widespread disease that causes significant health problems that may lead to death [7]. Diabetics are at an increased risk for developing emotional problems such as depression which may lead to a lower quality of life [8]. Type 2 diabetes mellitus is a metabolic disorder whose prevalence continues to dramatically increase around the globe. The IDF [1] estimated that 415 million adults worldwide have developed diabetes and, among this population, 318 million suffer from impaired glucose tolerance. By the year 2040, this number is expected to increase to 642 million adults with diabetes and 482 million people with impaired glucose tolerance [2]. The disease is reaching pandemic proportions and represents a significant burden on countries worldwide, especially poorly developed countries [2].

The American Diabetes Association [9] stated that the total diabetes cost for 2017 was $\$ 327$ billion, with $\$ 237$ billion in direct medical costs and $\$ 90$ billion in reduced productivity. Diabetes patients experience an average medical cost of $\$ 16,750$ per year, of which $\$ 9,600$ is allocated specifically to diabetes treatment [9]. The average medical expenses for these patients are approximately 2.3 times higher than non-diabetics. The elderly population (age 65 and older) has experienced an increase in diabetes prevalence and medical costs, contributing to the rising overall costs to health insurance programs [9].

Diabetes is associated with many challenges including adjusting to a new diagnosis, diabetes distress impairing self-management, psychological insulin resistance, and fear of hypoglycemia. Many individuals with depression have an approximately $40 \%$ to $60 \%$ increased risk of developing type 2 diabetes [10]. Depression has a negative influence on diabetes control such as binge eating, poor selfcare, a lack of sleep, persistent concerns about complications, and the need for continual monitoring and treatment of blood sugar. It is a significant reason why the intervention should be implemented. Many interventions such as lifestyle modification and pharmacological treatment have been evaluated to demonstrate that T2DM can be avoided or delayed in high-risk individuals with impaired glucose tolerance. Thorough regular lifestyle intervention for people with T2DM who are overweight or obesity reduces the risk of depressive symptoms by $15 \%$ [11].

\section{Theoretical Framework- Trans-Theoretical Model}

\section{Rationale}

The rationale for this project was based on two behavioral models. The trans-theoretical model of change describes the process individuals go through when contemplating and executing change. The health behavioral model examines individuals' attitudes and beliefs that inform health decisions and subsequent health behaviors. Each model is described in more detail in the following sections.

\section{Trans-theoretical model}

The basis for this DNP quality improvement project was DiClemente and Prochaska's [12] trans-theoretical model (TTM), or the stages of change model. The model is used by providers to help patients in all stages of their readiness to change (Figure 1). The importance of the process is not on the outcome or the relapse that can occur during the process; rather, the importance lies in the natural evolution that results in a healthier lifestyle as patients learn to manage their diabetes [12]. The trans-theoretical model was developed by Prochaska and DiClemente in the late 1970s and evolved through several research studies in which the researchers investigated the capabilities of chain smokers who quit smoking on their own in comparison to those who

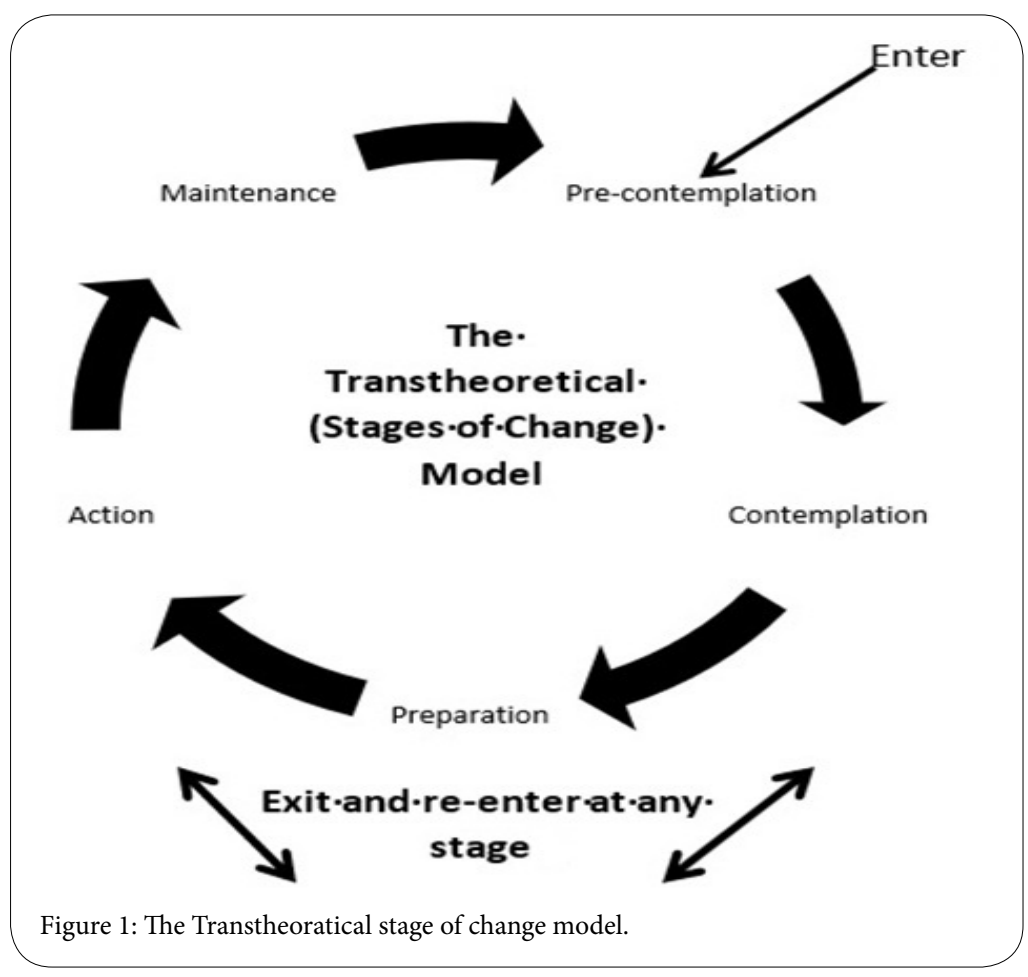


Citation: Adepoju GA (2020) Evaluation of a T2DM Lifestyle Modification Counseling Program to Improve Patient Engagement in Diabetes Self-Care in a Community Mental Health Clinic. Int J Nurs Clin Pract 7: 319. doi: https://doi.org/10.15344/2394-4978/2020/319

Page 3 of 12

required additional professional, therapeutic treatment. DiClemente and Prochaska sought to understand why some people were capable of quitting on their own.

The focus of the TTM is on the individual's decision-making and intention to change behavior. The TTM operates on the hypothesis that people do not change behavior quickly and authoritatively unless they are ready to change the behavior, especially routine behavior that occurs through a continuous process [13]. The process involves moving through different stages; individuals may relapse and start again from the beginning of the stage process [13]. The TTM served as a guide to improve the behavior intervention designed to achieve positive care outcomes for people who live with diabetes.

The TTM postulates that individuals move through six stages of change: pre-contemplation, contemplation, preparation, action, maintenance, and termination (Figure 1). The T2DM lifestyle modification moved the individual patient with diabetes through each stage of change. The DNP intervention strategies encouraged individuals with diabetes to adjust to a new behavior and then work through the model to maintenance, the ideal stage of behavior.

\section{Operational definition of terms}

\section{Diabetes}

Diabetes is a cluster of metabolic diseases that are characterized by abnormally high blood glucose levels. Patients with uncontrolled blood sugar are at a higher risk of developing severe health problems such as stroke or heart attack [14] (Figure 2).

\section{Screening for diabetes}

Screening for diabetes is an initial step in the identification and diagnosis of T2DM. Moreover, an awareness of the risk factors for diabetes in adults can guide screening. Predisposing risk factors for diabetes include age, body-mass index, waist circumference as measured below the ribs of men and women, and a family history of diabetes. The ADA [3] recommended screening for T2DM using an informal assessment of risk factors. However, validated tools should be considered for use with asymptomatic adults [3].

Testing for T2DM should be done for adults of any age who are overweight or obese (BMI $\geq 25 \mathrm{~kg} / \mathrm{m}^{2}$ or R BMI $\geq 23 \mathrm{~kg} / \mathrm{m}^{2}$ in Asian Americans) and who have one or more additional risk factors for diabetes. If tests are routinely scheduled, repeat testing should be done in three-year intervals at a minimum. According to the ADA [3] recommendations include fasting plasma glucose collection, 2-hour plasma glucose test, and the A1C test as equally appropriate. In patients with diabetes, the guidelines recommend identifying and treating other cardiovascular disease risk factors [3]. One point of concern is that T2DM is among the leading causes of death in the United States and is associated with both microvascular and macrovascular complications [3].

\section{Treatment guidelines}

There are many clinical guidelines for the treatment of T2DM available to providers in a clinical setting. The project discussed in this paper employed the American Association of Clinical Endocrinologists (AACE) and the American Diabetes Association (ADA) guidelines [6]. Glycated hemoglobin (HbA1c) is expressed as the percentage of hemoglobin that is glycated and is widely used to measure the efficacy of diabetes treatment and as a basis for therapeutic adjustments [6].

\section{Depression}

Depression is a cluster of behavioral problems characterized by the loss of interest in activities that were previously enjoyable to the patient, mood swings, and other cognitive, physical, and behavioral symptoms [15].

\section{Depression screening}

Predisposing risk factors for depression include previous bouts of depression, vascular problems, diabetes, significant physical and chronically disabling illness, social disadvantage, and low social

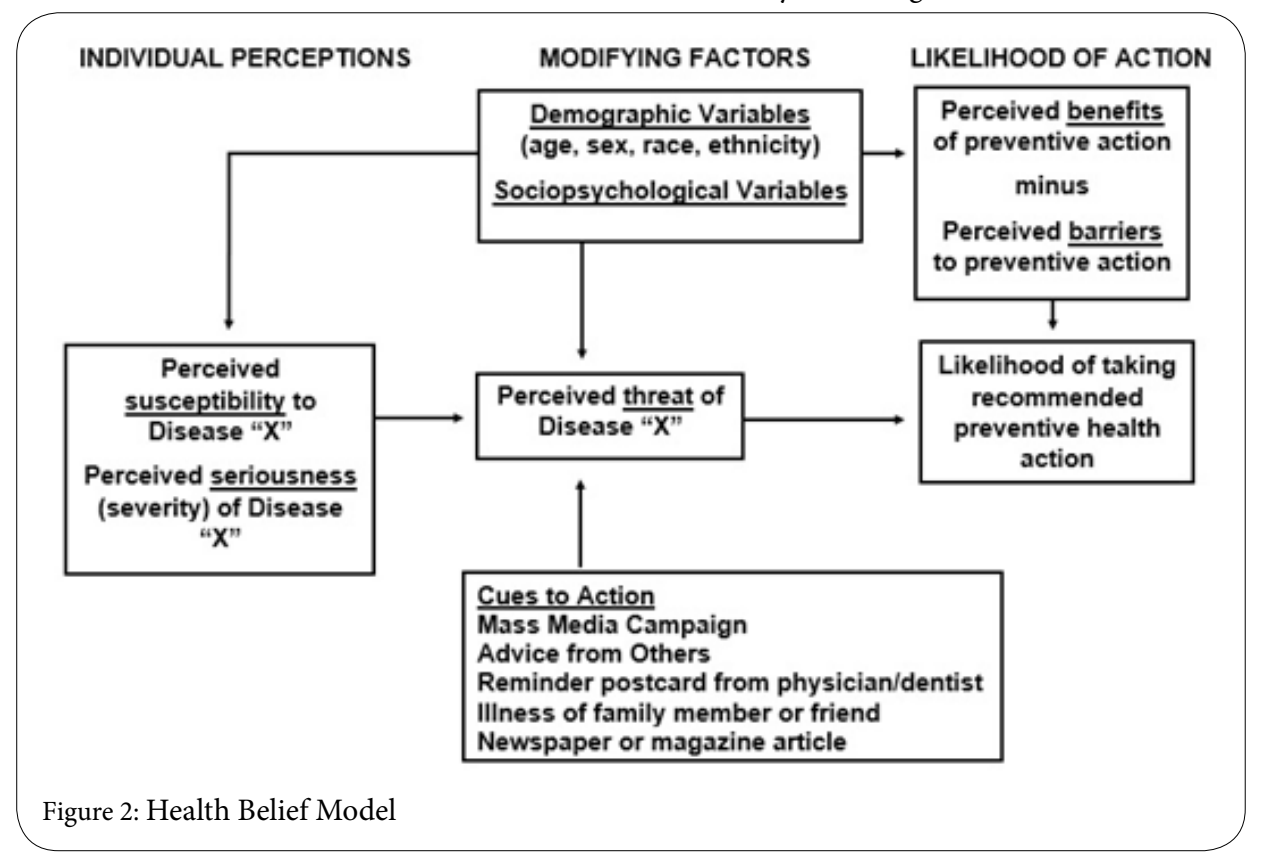


support. Criteria for major depression include a significant change in appetite or weight, sleep disturbances, psychomotor agitation or retardation, loss of energy or fatigue, guilt or feelings of worthlessness, poor concentration or decision making, and continuous thoughts of death or suicide. The patient must meet five of these criteria to be diagnosed with major depression [16].

The purpose the T2DM lifestyle modification project was to increase participants' care engagement to improve their medication and selfmanagement skills, increase their physical activity, and improve their dietary habits. The increased risk of major depression in patients with diabetes and depression has a significant negative impact on the quality of life of individuals with diabetes [17]. Depression is also linked to poorer glycemic control, worse cardiovascular outcomes, and an upsurge in healthcare consumption [18]. The data collected allowed the DNP project leader to assess whether brief lifestyle modification counseling provided by a nurse practitioner (NP) could impact the self-management of diabetes among T2DM patients at one community mental health center. The PAM13 assessment was administered during the pre-implementation phase to determine patients' understanding of T2DM self-management as experienced by participants at the CMHC.

The DNP administered the PAM13 again at the conclusion of the implementation phase to determine if participants reported a greater understanding and willingness to self-manage their diabetes. The objective of this quality improvement project was to investigate whether brief lifestyle modification counseling could improve the level of patient self-management practiced by T2DM adult patients at the CMHC.

The project employed a retrospective chart review and concurrent chart review designed to determine whether the intervention was effective. The retrospective chart review is commonly and appropriately used for study techniques by healthcare disciplines as a means to direct subsequent prospective investigations [19]. For both pre- and post-implementation of the project, the DNP project leader reviewed each patient's chart for six weeks. The descriptive statistical analysis provided an appropriate study design to measure the effectiveness of the intervention in achieving the desired outcome of this objective and goal. Furthermore, the presentation of raw data is difficult to visualize, mainly when a large amount of data is obtained, making statistical processes suitable for this project [20].

The DNP project leader used a Wilcoxon signed-rank test for this project. A Wilcoxon signed-rank test is the nonparametric test equivalent to the dependent $\mathrm{t}$-test [21]. The test is used to compare two sets of scores that come from the same participants [21]. This test was appropriate for the DNP project. Three assumptions of the Wilcoxon signed-rank test are required for the project to produce a valid result. The assumptions are: (a) the dependent variables in the study must be measured as ordinal or continuous level, (b) the dependent variable in the study should consist of two categorical "related groups" or "matched pairs," and (c) the distribution of the difference between the two related groups should be symmetrical in shape [21].

The Wilcoxon signed-rank statistical method was selected for this project since the independent variable, engagement in the management of care was in ordinal form and the dependent variable, improving scores on the PAM13, was categorical with related samples [22]. The third assumption's condition is that the distributions between the two related groups are symmetrical in shape. This condition was met with the pre- and post-test scores from the same group [21].
The sample size was 62 patients to be statistically significant. The sample size determined if there was a statistical significance in the difference between the results due to the counseling program or if the differences could have occurred by chance [20]. Quantitative scoring was used to prove or disprove the null hypothesis: there is no difference in patients' self-management skills based on the PAM13 between pre- and post-assessment scores. The DNP used tables and graphs to display the results of the data analysis [22].

\section{Measures}

The DNP measured the T2DM patients' engagement with care using the validated PAM13. The PAM13 is used to measure patient self-management skills such as patients' knowledge of their disease, skill to self-manage their disease, self-confidence in their abilities to manage their disease, self-efficacy in self-managing behavior, and readiness to change health-related behaviors. Four stages indicate different levels of patient activation or readiness to self-manage. The PAM13 assumes that patients progress through the four stages as they become activated. The PAM13 assessment, developed by Hibbard and Greene [23], consists of the following four different level of activation:

1. Level 1: Belief questions assess the participants' belief that their active role is important in their health and taking an active role in their own health care is the most important factor in determining their health and ability to function.

2. Level 2: Confidence questions assess the patients' level of confidence and knowledge to act on their own behalf.

3. Level 3: Action questions assess the patients' ability to take action.

4. Level 4: Stay the course questions assess the patients' ability to stay engaged under stress (see Appendix D).

The PAM13 consist of four stages: the first stage consists of two questions; the second stage consists of six questions; the third stage consists of three questions, and the fourth stage consists of two questions. The PAM13 utilizes a scoring key with a 5-point Likert-type scale with values ranging from "strongly disagree" to "strongly agree" [22]. Hibbard and Greene [22] conducted a study in an experimental setting with the PAM13 incorporating a longitudinal design and the use of scientifically validated measures for the assessment of patient activation. Hibbard and Greene evaluated the effect access to a patient portal had on patient activation among chronically ill patients. Hibbard and Greene also examined the relationship between PAM13 and the temporal proximity of severe diabetes and found that patients starting at a lower level of activation showed a superior improvement in activation over patients whose last severe diagnosis was made less than three years prior. The study's findings demonstrated that the intervention had the most significant effect on patients beginning at the highest level of patient activation [24]. Harvey, Briggs Fowles, $\mathrm{Xi}$, and Terry [25] evaluated whether changes in patient responses on the PAM are related to changes in health status and health behaviors. Harvey et al. found that a positive change in health behaviors at all levels is associated with improved PAM scores, mostly at Level Four. Individuals are capable of making major changes at every level of PAM. Harvey et al. recommended that interventions be designed to encourage movement from lower levels of PAM to higher levels activation.

Hibbard and Greene [22] reviewed the patient activation measures make to the skill and confidence of the patient seeking to become 
actively engaged in their health care. The study also involved evaluating health costs, patient experiences, and health outcomes. Hibbard and Greene found that patients who are more activated enjoy better outcomes and care experiences. Evidence also showed that activation impacts healthcare costs [22]. The interventions that tailor support to the individual's level of activation and build skills and confidence are effective in increasing patient activation. Furthermore, the point at which the patient enters the intervention (level of PAM) determines the improvement experienced in the self-management of their care. Hibbard and Greene recommended that policies and interventions aimed at strengthening the patient's role in managing their health care can contribute to improved outcomes. Instrument reliability was determined using a test-retest measure. According to Hibbard and Greene, the PAM13 is the most widely used instrument all over the world for health-related diabetes care. The PAM13's education guidelines are universally used as survey instruments today in several countries such as Greece, Ireland, Jamaica, Malaysia, New Zealand, Northern Ireland, Norway, and Singapore. The test can be integrated into the automated medical record. It is a highly reliable instrument with satisfactory construct validity, a short and generic measure of diabetes care, and an education guideline. The PAM13 scale was quick, flexible, and more comfortable to use for this DNP project. Permission to use the PAM13 was obtained through purchase of the instrument from Insignia Health (see Appendix E).The DNP performed a retrospective chart review for the six week window (December 1, 2018 through January 30,2019$)$ prior to implementation of the lifestyle modification counseling program, and a concurrent chart review continued for six weeks during program implementation (February 1, 2019 through April 1, 2019). The sample size was estimated at 62 consenting participants. A total of 73 participants were recruited and participated in both the pre- and post-interventions. The DNP project leader completed the retrospective chart review and concurrent chart review for both pre- and post-time periods to reduce data recording errors. Sample size was determined from the DNP caseload. To ensure completeness of the data collection, the DNP project leader used an Excel spreadsheet to code data related to appointments, patient insurance, age, gender, ethnicity, and diagnoses.

\section{Analysis}

Independent and dependent variables play an essential role when conducting quantitative research. The independent variable is responsible for the effect on the dependent variable[26]. The DNP used the Wilcoxon signed-rank test for this project. A Wilcoxon signed-rank test is the nonparametric test equivalent to the dependent t-test [21]. The Wilcoxon signed-rank test is used to compare two sets of scores that come from the same participants [21].

The DNP selected the statistical method for this project since the independent variable, engagement in the management of care was in ordinal form and the dependent variable, improving scores on the PAM, was ordinal with related samples [22]. The third assumption's condition was that the distributions between the two related groups were symmetrical in shape. This condition was met with the pre- and post-test scores from the same group [21].

\section{Ethical Considerations}

According to the EBP project, multiple ethical and legal issues must be addressed by the researchers in the project including the respect for privacy and confidentiality, beneficence, and justice. Ethical considerations in research are fundamental concerns because they guide the researcher's actions throughout the study and help to discern between acceptable and unacceptable behaviors [27]. Furthermore, ethical considerations are critical to data sharing, copyright guidelines, confidentiality, and human rights [27].

The use of human subjects is one of the most significant moral concerns of our era. The magnitude of these ethical implications has led the majority of research organizations and clinical institutions to form institutional review boards (IRB). Institutional review boards are the organizations tasked with assisting and protecting a patient's human rights throughout the course of a trial. The IRB also shelters institutions and the researchers from possible legal ramifications stemming from any unacceptable behavior [27].

The primary points of concern addressed by this project design included fair subject selection; independent review of the study to eliminate conflicts of interest; and the protection of subjects' privacy, interests, and welfare [27]. Each participating subject signed an informed consent form asserting that they understood the researcher's explanation of the benefits, side effects, and procedures of the study. If these critical components are not fully observed, the project can generate serious legal problems for the researcher [27].

The Wilmington University Human Subject Review Committee reviewed and approved the project as described. The DNP requested and received permission to conduct the project at the program site. Each participant completed an informed consent form and signed it in the presence of the DNP after both the form and the program were thoroughly explained, and the participant confirmed their comprehension of the process. The DNP completed certification in the protection of human research subjects. The DNP met with each participant every two weeks. All information was entered into the DNP laptop and was accessible only by the DNP with password for protection. The records will be maintained for five years based on federal regulations. Data are anonymous and no patient identifiers are recorded. During the first session of lifestyle modification counseling, therapy took place between the DNP and the participant. The DNP talked with the participant about food, exercise, and medication adherence. The DNP also provided a follow-up telephone number to the participant and information about a diabetes information group to answer any questions and provide clarification of provided information. The participants completed four sessions over the twomonth period, at an interval of every two weeks.

\section{Summary}

The incidence of diabetes mellitus in the United States is multifactorial. These factors include an inconsistency in teaching material that is tailored to patients' culture or individual needs, a limited time to teach patients regarding diabetes care, a trained primary care provider, or education that is not patient-centered. Furthermore, a lack of family or staff support is increasingly cited as a factor that contributes to a patient's inability to manage diabetes. Each of these factors, however, leads to poor clinical outcomes which result in uncontrolled T2DM.

The participants in this DNP project were currently enrolled in the CMHC program and had diagnoses of T2DM and depression identified on the electronic medical record (EMR). The CMHC where this project took place currently lacks a program to screen and manage such patients. The DNP student administered the PAM13 
Citation: Adepoju GA (2020) Evaluation of a T2DM Lifestyle Modification Counseling Program to Improve Patient Engagement in Diabetes Self-Care in a Community Mental Health Clinic. Int J Nurs Clin Pract 7: 319. doi: https://doi.org/10.15344/2394-4978/2020/319

Page 6 of 12

assessment during the pre-implementation phase to determine patients' understanding regarding T2DM self-management. The DNP student administered the PAM13 assessment again at the conclusion of implementation phase to determine if diabetes patients reported a greater understanding and willingness to self-manage their diabetes.

\section{Sample Characteristics}

New Beginnings Community Mental Health Center is one of several Samaritan Daytop Village outpatient mental health centers. Samaritan Daytop Village has been improving the quality of life for New Yorkers facing adversity for more than 50 years. The program is nationallyrecognized for their work in the treatment of substance use disorder, specialized services for veterans, and services for homeless individuals and families. Samaritan Daytop Village offers a continuum of services that includes health care, mental health care, addiction treatment, transitional and permanent housing, education and employment services, care coordination, and services for adolescents and seniors. Annually, Samaritan Daytop Village serves over 28,000 people at more than 50 facilities throughout New York City, Long Island, and upstate New York, and they continue to grow [28]. New Beginnings Community Mental Health Center provided the setting for the DNP project.

Before project implementation, the DNP project leader reviewed patient charts for patient laboratory results, self-report of diabetes diagnosis, health history, and medication history for approximately six weeks. Patients with co-occurring diabetes and depression were identified for the project. Upon obtaining informed consent, the DNP project leader administered the PAM13 questionnaire to participants to assess and identify participants' engagement with their diabetes care. The DNP project leader used the PAM13 to measure patient self-management skills such as assesses a patient's knowledge of their disease, skill to self-manage their disease, self-confidence in their abilities to manage their disease, self-efficacy in self-managing behavior, and readiness to change health-related behaviors.

The participants were currently enrolled in the CMHC program and had diagnoses of T2DM and depression identified on the electronic medical record (EMR). The DNP selected every patient who met the inclusion criteria until at least 73 participants consented to participate in the project. It was essential to interview the patients to understand their beliefs concerning the cause of diabetes or the development of the disease. It was also crucial to inquire how participants to proceed with their treatment and lifestyle modification, including the addition of follow-up visits with their PCP and endocrinologist and the inclusion of stress management techniques in their daily routine.

A total of 73 patients participated in both the pre- and postinterventions. The average age was $59.36(\mathrm{SD}=8.739)$ years old with the youngest participant being 40 years old and the most senior participant being 78 years old for an age range of 38 years. As seen in Table 1, the participants were mostly female $(\mathrm{n}=52,71.2 \%)$, Hispanic $(\mathrm{n}=59,80.8 \%)$ and were diagnosed with depression $(\mathrm{n}=39,53.4 \%)$.

\section{Results}

A Wilcoxon signed-rank test was used for this project. A Wilcoxon signed-rank test is the nonparametric test equivalent to the dependent t-test [21]. The test is used to compare two sets of scores that come from the same participants [21]. This test was appropriate for the DNP project. The three assumptions of the Wilcoxon signed-rank

\begin{tabular}{|l|l|l|}
\hline Item & Frequency & Percent \\
\hline Gender & & \\
\hline Female & 52 & 71.2 \\
\hline Male & 21 & 28.8 \\
\hline Total & 73 & 100.0 \\
\hline Race & & \\
\hline Black & 7 & 9.6 \\
\hline Hispanic & 59 & 80.8 \\
\hline White & 7 & 9.6 \\
\hline Total & 73 & 100.0 \\
\hline Mental Health Diagnosis & & \\
\hline Depression & 39 & 53.4 \\
\hline Depression/Anxiety & 25 & 34.2 \\
\hline Depression/Anxiety/Bipolar & 7 & 9.6 \\
\hline Depression/Anxiety/Schizophrenia & 2 & 2.7 \\
\hline Total & 73 & 100.0 \\
\hline Table 1: Participant Demographics. & & \\
\hline
\end{tabular}

test were required for the project to produce a valid result. The assumptions were as follows: (a) the dependent variables in the study were measured as ordinal or continuous level, (b) the dependent variable in the study consisted of two categorical "related groups" or matched pairs," (c) the distribution of the difference between the two related groups was symmetrical in shape.

This statistical method was selected for this project since the independent variable, engagement in the management of care, was in ordinal form and the dependent variable, improving scores on the PAM, was ordinal with related samples [22]. The third assumption's condition was that the distributions between the two related groups were symmetrical in shape. This condition was met with the pre- and post-test scores from the same group [21].

The Wilcoxon signed rank test was used and a statistically significant grouped median difference of 3.83 was found between the pre and post test scores, $z=-4.070, \mathrm{p}<0.001$ (Table 2). This means the intervention had a significant impact in raising the PAM scores and supported the PICOT question This DNP project, therefore, empowered patients to engage in self-management of their diabetes through its counseling program (Figure 3).

\begin{tabular}{|c|c|c|c|c|c|c|}
\hline \multirow[b]{2}{*}{ Outcome } & \multicolumn{3}{|c|}{ Median } & \multirow[b]{2}{*}{$\begin{array}{l}\text { Median } \\
\text { Difference }\end{array}$} & \multirow[b]{2}{*}{$z$} & \multirow[b]{2}{*}{$p$} \\
\hline & $\begin{array}{l}\text { Pre- } \\
\text { Test }\end{array}$ & $\begin{array}{l}\text { Post- } \\
\text { Test }\end{array}$ & $n$ & & & \\
\hline PAM13 & 42.03 & 45.86 & 73 & -3.83 & -4.070 & $.000^{*}$ \\
\hline
\end{tabular}

Table 2: Descriptive Statistics and Wilcoxon Signed-Rank Results for PAM.

${ }^{*}$ significant at the .01 level.

\section{Data Analysis Plan}

The DNP student utilized SPSS for statistical analysis. The initial data analysis plan was conducted in three phases. First, all project variables were presented using descriptive statistics such as mean, standard deviation, and minimum/maximum values for continuous variables (interval/ratio level) and frequencies and percentages for categorical variables (nominal/ratio level). 


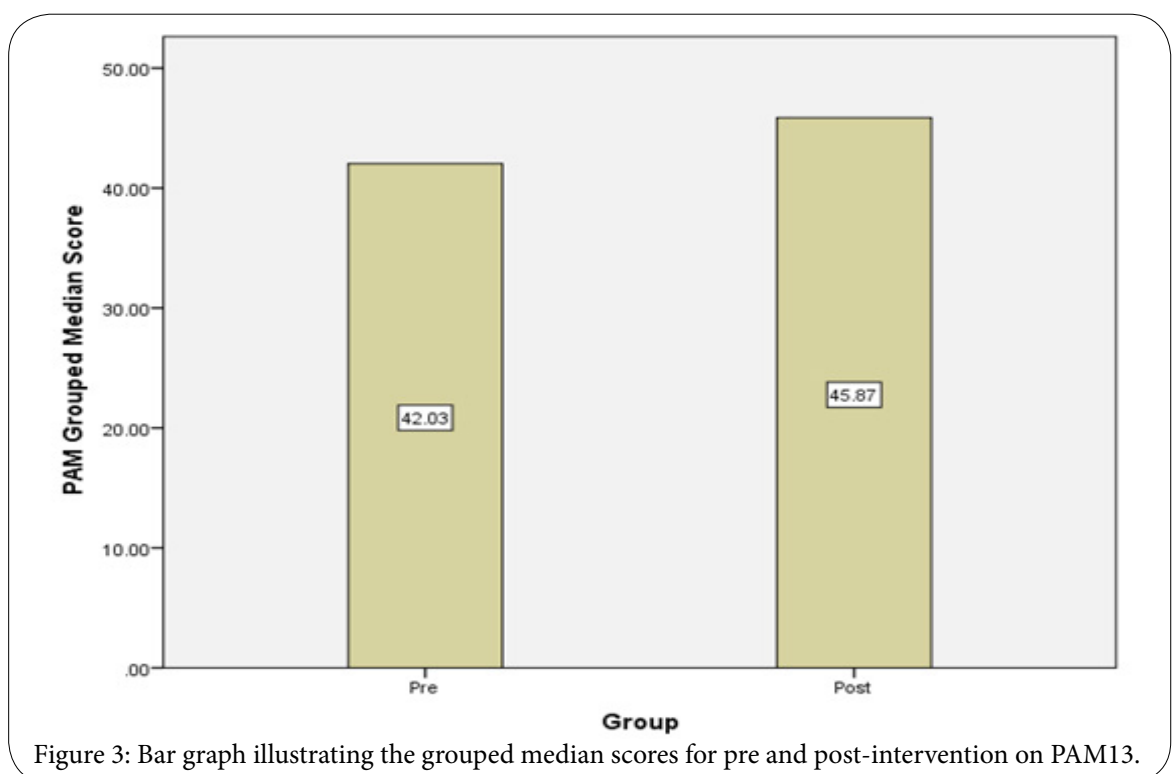

Next, a series of bivariate tests were used to produce inferential findings. Specifically, a paired-samples t-test was used to identify if matched pretest to posttest PAM scores changed at a statistically significant level $(\mathrm{p}<.05)$. Additionally, pretest to posttest difference scores were computed through subtracting pretest scores from respective posttest scores. Bivariate tests were then used to identify if pre/post change scores were related to study participant age (via Pearson's $r$ correlation), as well as gender (via independent-samples t-test) at a statistically significant level.

Third, if gender or age evidenced a statistically significant relationship with pretest to posttest PAM difference scores, a multivariate model, specifically a repeated measures general linear model, was used to model pretest to posttest change in matched paired dependent variable scores while controlling for the effect of the demographic factor. If these factors were not related to pretest to posttest change, the bivariate paired samples t-test was used as the final inferential finding.

A second analysis was also conducted to examine changes in PAM level from pretest to posttest. Specifically, chi-square analysis determined if the percentage of project participants at level 1 at pretest was significantly different than the percentage at level 1 at posttest. Additionally, bivariate analysis determined if changes from level 1 to greater levels from pretest to posttest were related to study participant age or gender.

Within the final inferential analysis presented, the parametric test assumptions of normality and no undue influence of outlier scores were examined and revealed no significant problems. Regarding the psychometric properties on the PAM instrument, a reliability analysis indicated sufficient levels of internal consistency (Cronbach's Alpha > $.70)$ for PAM scores at pretest (Cronbach's Alpha $=.87)$ and posttest (Cronbach's Alpha $=.93)$.

Regarding missing data, two project participants did not provide data for one item on the PAM survey. The mean of the valid responsesprovided by the other project participants were imputed in the missing cells for those items to address the missing data points. One participant did not report their gender. This data point was left missing. There were no other missing data in the dataset.
In terms of statistical power for the paired-samples t-test, the $G^{*}$ power software indicated that a medium size effect (Cohen's $d=.50$ ) between the related means within the paired-samples t-test (2-tailed) with power set at .80 and alpha set at .05 , would require a sample size of 34 study participants. Thus, the sample of 72 study participants provided sufficient statistical power for the analysis.

\section{Descriptive Analysis}

Table 3 presents a descriptive analysis of project participant's gender and PAM levels at pretest and posttest. Data indicated that the sample was about three-quarters female $(\mathrm{n}=51 ; 71.8 \%)$. The majority of the sample was at PAM level 1 at pretest $(n=62,86.1 \%)$, but only about half at posttest $(\mathrm{n}=39,54.2 \%)$. Regarding pretest to posttest PAM level improvement from 1 , about half $(\mathrm{n}=33,45.8 \%)$ evidenced a change Yes (where pretest scores $=1$ and posttest scores $=>1$ or pretest scores are $>1)$ and the remainder $(n=30,54.2 \%)$ were coded as No (where pretest $=1$ and posttest $=1$ ).

\begin{tabular}{|c|c|c|}
\hline Variable & $\mathrm{n}$ & $\%$ \\
\hline \multicolumn{3}{|l|}{ Gender } \\
\hline Female & 51 & 71.8 \\
\hline Male & 20 & 28.2 \\
\hline \multicolumn{3}{|l|}{ Pre-PAM Level } \\
\hline 1 & 62 & 86.1 \\
\hline 2 & 8 & 11.1 \\
\hline 3 & 1 & 1.4 \\
\hline 4 & 1 & 1.4 \\
\hline \multicolumn{3}{|l|}{ Post-PAM Level } \\
\hline 1 & 39 & 54.2 \\
\hline 2 & 21 & 29.2 \\
\hline 3 & 7 & 9.7 \\
\hline 4 & 5 & 6.9 \\
\hline \multicolumn{3}{|c|}{ Pre-Post PAM Level Improvement from 1} \\
\hline Yes $($ pre $=1 \&$ post $=>1 /$ pre $=>1)$ & 33 & 45.8 \\
\hline No $($ pre $=1 \&$ post $=1)$ & 39 & 54.2 \\
\hline
\end{tabular}

Table 3: Descriptive Analysis of Categorical Demographic Characteristics $(\mathrm{N}=72)$. 
Citation: Adepoju GA (2020) Evaluation of a T2DM Lifestyle Modification Counseling Program to Improve Patient Engagement in Diabetes Self-Care in a Community Mental Health Clinic. Int J Nurs Clin Pract 7: 319. doi: https://doi.org/10.15344/2394-4978/2020/319

Page 8 of 12

Table 4 presents a descriptive analysis of age, PAM pretest, PAM posttest, and PAM change scores. Data indicated that the average study participant was $59.22(\mathrm{SD}=8.74, \mathrm{MIN} / \mathrm{MAX}=40.00-78.00)$ years old. The average pretest score was 43.03 ( $\mathrm{SD}=6.35$, MIN/MAX $=26.10-77.70)$ and posttest scores was $49.98(\mathrm{SD}=13.05, \mathrm{MIN} / \mathrm{MAX}$ $=38.10-100.00$ ). Thus, the average study participant (via the pre/post difference score) evidenced a change of 6.95 ( $\mathrm{SD}=11.52$, MIN/MAX $=-8.90-48.50)$ from pretest to posttest.

Figure 4 is a histogram depicting the distribution of pretest to posttest PAM change scores. Note there are eight outlier scores to the right side of the distribution. When the eight outlier pretest to posttest change scores were removed, the distribution of all the scores was approximately normal (as the skewness and kurtosis were not approximately two times each respective standard error of each). The inferential findings within the remainder of the study did not differ in terms of statistical significance when outlier scores were removed, so the analysis retained those values (as the outliers did not have an undue influence of the findings).

\section{Bivariate Analysis}

Table 5 presents a Pearson's $r$ correlation examining the relationship between age and PAM pre/post difference scores. The 2-tailed correlation indicated that age was not related to PAM pre/post difference scores at a statistically significant level, $\mathrm{r}(70)=-.14, \mathrm{p}=.23$.

\begin{tabular}{|c|c|c|}
\hline Variable & Age & PAM Pre/Post Difference \\
\hline Age & -- & $-.14^{1}$ \\
\hline PAM Pre/Post Difference & & -- \\
\hline
\end{tabular}

Table 6 presents an independent-samples t-test analysis of PAM pretest to posttest change scores by gender. Data indicated that pre/ post change scores were not significantly related to gender, $\mathrm{t}(69)=$ $.23, \mathrm{p}=.82$.

\begin{tabular}{|l|l|l|l|l|l|l|}
\hline Variable & $n$ & $\%$ & $S D$ & $t / F$ & $d f$ & $p$ \\
\hline Gender & & & & .23 & 69 & .82 \\
\hline & Female & 51 & 6.82 & 12.04 & & \\
\hline & Male & 20 & 7.53 & 10.60 & & \\
\hline
\end{tabular}

Table 6: Independent Samples t-test Analysis of PAM Pre/Post Change Scores by Gender $(\mathrm{N}=71)$.

Table 7 presents a paired-samples t-test examining the changes in pretest to posttest PAM scores. Bivariate analysis indicated that pretest to posttest changes in mean PAM scores did achieve statistical significance, $\mathrm{t}(71)=-5.12, \mathrm{p}<.001$. Analysis indicated a medium/ large Cohen's d effect size of -0.62 .

Figure 5 is a graph depicting pretest to posttest PAM scores.

\begin{tabular}{|c|c|c|c|c|c|c|c|}
\hline \multirow[t]{2}{*}{ Variable } & \multirow[t]{2}{*}{ M } & \multirow[t]{2}{*}{ SD } & \multirow[t]{2}{*}{ MIN/MAX } & \multicolumn{2}{|c|}{ Skewness } & \multicolumn{2}{|l|}{ Kurtosis } \\
\hline & & & & Statistic & Std. Error & Statistic & Std. Error \\
\hline Age & 59.22 & 8.74 & $40.00 / 78.00$ & 0.04 & .28 & -0.26 & .56 \\
\hline Pre-Test Score & 43.03 & 6.35 & $26.10 / 77.70$ & 2.50 & .28 & 12.62 & .59 \\
\hline Post-Test Score & 49.98 & 13.05 & $38.10 / 100.00$ & 2.06 & .28 & 4.44 & .56 \\
\hline Pre/Post Difference & 6.95 & 11.52 & $-8.90 / 48.50$ & 1.98 & .28 & 3.97 & .56 \\
\hline
\end{tabular}

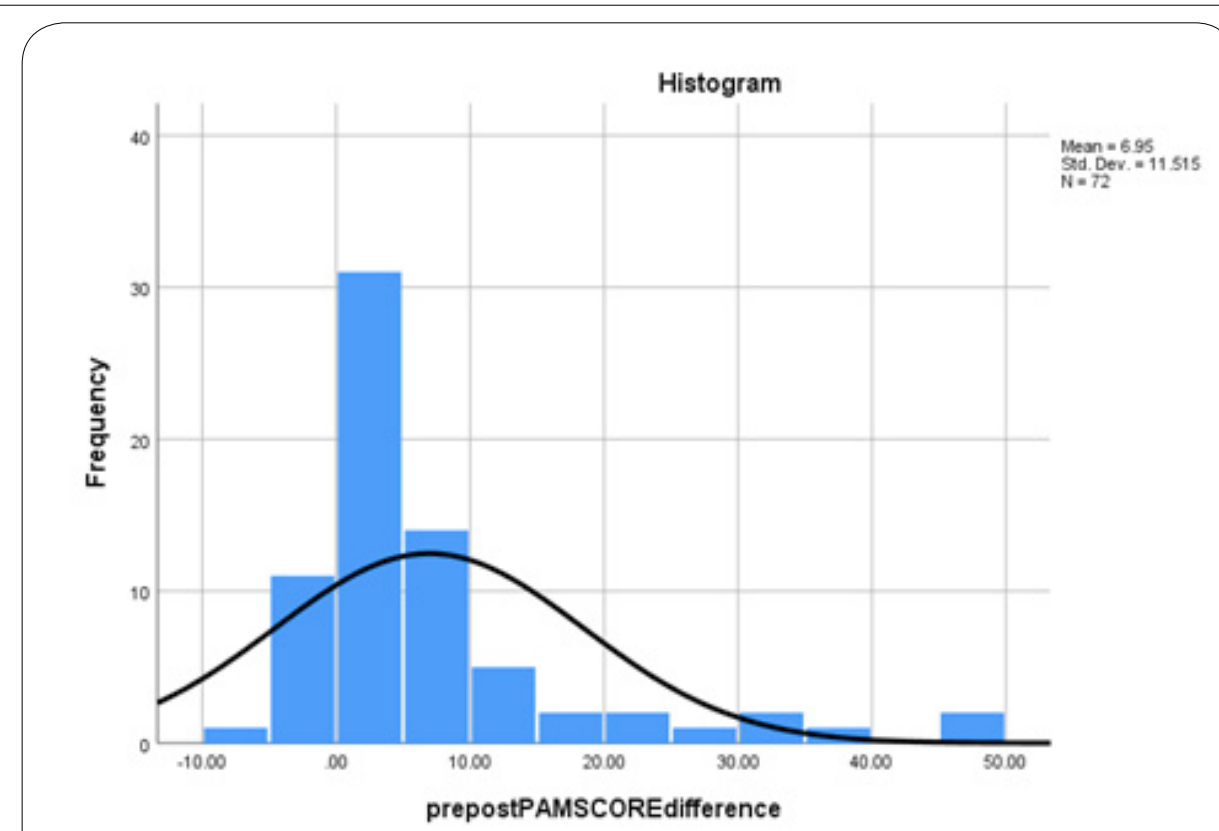

Figure 4: Distribution of Pretest to Posttest PAM Change Scores $(\mathrm{N}=72)$. 
Citation: Adepoju GA (2020) Evaluation of a T2DM Lifestyle Modification Counseling Program to Improve Patient Engagement in Diabetes Self-Care in a Community Mental Health Clinic. Int J Nurs Clin Pract 7: 319. doi: https://doi.org/10.15344/2394-4978/2020/319

Page 9 of 12

\begin{tabular}{|c|c|c|c|c|c|c|}
\hline Timepoint & $\mathrm{n}$ & M & SD & $\mathrm{t}$ & $\mathrm{df}$ & $\mathrm{p}^{*}$ \\
\hline & & & & -5.12 & 71 & .000 \\
\hline Pretest & 72 & 43.03 & 6.35 & & & \\
\hline Posttest & 72 & 49.98 & 13.05 & & & \\
\hline
\end{tabular}

Table 8 presents a chi-square analysis of greater than PAM level 1 vs. $>1$ by pretest and posttest time points. Data indicated that the percentage of study participants greater than level 1 at pretest $(\mathrm{n}=$ $10,13.9 \%)$ was significantly lower relative to the percentage of study participants greater than level 1 at posttest $(\mathrm{n}=33,45.8 \%), \mathrm{X}^{2}(1)=$ $17.54, \mathrm{p}<.01$. The phi effect size for this relationship waslarge $(\mathrm{phi}=.35)$.

\begin{tabular}{|l|l|l|l|l|l|l|l|}
\hline \multicolumn{7}{|c|}{ PAM Level 1 } & \multicolumn{2}{|l|}{ PAM Level > 1 } & & & \\
\hline Variable & $n$ & $\%$ & & $n$ & $\%$ & $X^{2}(d f)$ & $p$ \\
\hline $\begin{array}{l}\text { Greater } \\
\text { than PAM } \\
\text { Level 1 }\end{array}$ & & & & & & $17.54(1)^{1}$ & .000 \\
\hline Pretest & 62 & 86.1 & & 10 & 13.9 & & \\
\hline Posttest & 39 & 54.2 & & 33 & 45.8 & & \\
\hline
\end{tabular}

Table 8: Chi-Square Analysis of $>$ PAM Level 1 vs. $>1$ by Time points $(\mathrm{N}=72)$. 1phi effect size $=.35$
Figure 6 is a chart illustrating the percentage of study participants at PAM Level 1 and greater than PAM level 1 at pretest and posttest.

Table 9 presents a chi-square analysis of pretest to posttest PAM level improvement from 1 by gender. Data indicated that the percentage of study participants that made this positive change was related to gender, $\mathrm{X}^{2}(1)=.00, \mathrm{p}=.99$.

\begin{tabular}{|l|l|l|l|l|l|l|l|}
\hline & \multicolumn{2}{|l|}{$\begin{array}{l}\text { No } \\
\text { (pre = 1; } \\
\text { post = 1) }\end{array}$} & \multicolumn{2}{l|}{$\begin{array}{l}\text { Yes } \\
\text { Pre = 1 \& Post } \\
>1 \text { or Pre = 1) }\end{array}$} & & & \\
\hline Variable & $n$ & $\%$ & & $n$ & $\%$ & $X^{2}(d f)$ & $p$ \\
\hline Gender & & & & & & $0.00(1)$ & .99 \\
\hline & Female & 28 & 54.9 & & 23 & 45.1 & \\
\hline & Male & 11 & 55.0 & & 9 & 45.0 & \\
\hline
\end{tabular}

Table 9: Chi-Square Analysis of Pretest to Posttest PAM Level Improvement from 1 by Gender $(\mathrm{N}=71)$.

Table 10 presents an independent-samples t-test indicating that PAM pretest to posttest change in PAM Level Improvement from 1 was not significantly related to age, $\mathrm{t}(70)=.06, \mathrm{p}=.95$.

\section{Summary}

The Wilcoxon signed rank test was selected for this project since the independent variable, engagement in the management of care, was

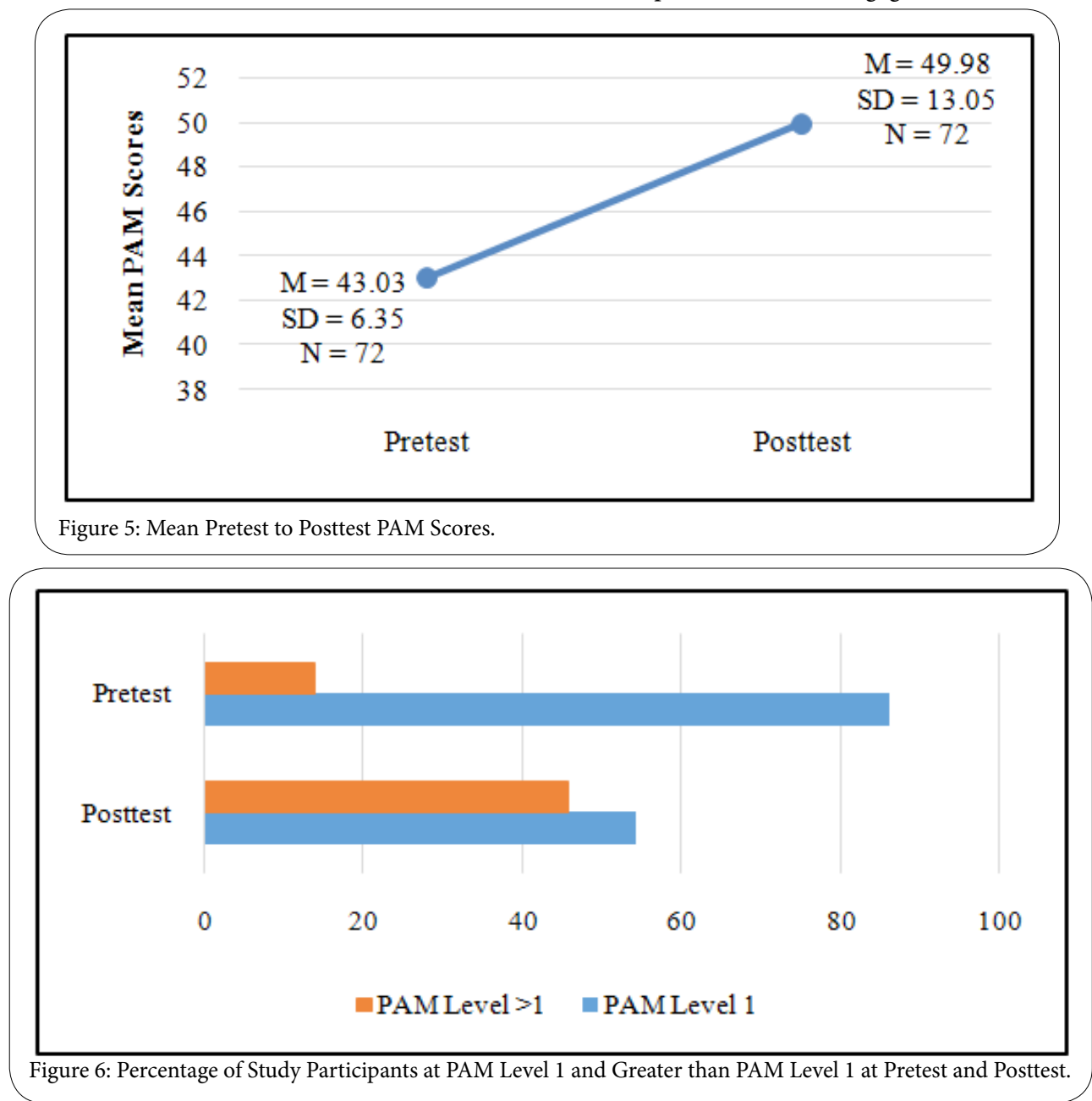


in ordinal form and the dependent variable, improving scores on the PAM, was ordinal with related samples [22]. The third assumption's condition was that the distributions between the two related groups were symmetrical in shape. This condition was met with the pre- and post-test scores from the same group [21].

\begin{tabular}{|l|l|l|l|l|l|l|}
\hline Variable & $n$ & $M$ & $S D$ & $t$ & $d f$ & $p$ \\
\hline $\begin{array}{l}\text { Pre/Post PAM Level } \\
\text { Improvement from 1 }\end{array}$ & & & & .06 & 70 & .95 \\
\hline $\begin{array}{l}\text { Yes (Pre = 1 \& Post > } \\
1 \text { or Pre = 1) }\end{array}$ & 33 & 59.15 & 8.70 & & & \\
\hline $\begin{array}{l}\text { No (Pre = 1 \& Post } \\
=1)\end{array}$ & 39 & 59.28 & 8.88 & & & \\
\hline
\end{tabular}

Table 10: Independent Samples t-test Analysis of Pre/Post PAM Level Improvement from 1 by age $(\mathrm{N}=72)$.

This statistical method was run and a statistically significant grouped median difference was found between the pre- and post-test scores. This means the intervention had a significant impact in raising the PAM13 scores and supported the PICOT question.

\section{Discussion and Implications}

\section{Summary}

The introduction of a lifestyle modification counseling program in a CMHC resulted in improved patient engagement in personal diabetic care. Median patient test scores on the PAM13 increased from 42.03 (pre-test) to 45.87 (post-test), a significantly significant difference at the .01 level, demonstrating intervention success. The Wilcoxon signed rank test was run and a statistically significant grouped median difference of 3.83 was found between the pre- and post-test scores, $\mathrm{z}=$ $-4.070, \mathrm{p}<0.001$. This means the intervention had a significant impact in raising the PAM scores and supported the PICOT question.

The PAM13's four levels indicated that patients were confident and willing to self-manage their diabetes. The lifestyle modification counseling concept places the patient and family at the center of health care. The patient becomes actively involved in their own care.

The basis for this DNP quality improvement project was DiClemente and Prochaska's [12] transtheoretical model, or the stages of change model. The model is used by DNP students to help patients in all stages of their readiness to change. The importance of the process is not on the outcome or the relapse that can occur during the process; rather, the significance lies in the natural evolution that results in a healthier lifestyle as patients learn to manage their diabetes [12].

The DNP intervention strategies encouraged individuals with diabetes to adjust to a new behavior and then work through the model to maintenance, the ideal stage of behavior. The patients were the major players to facilitate change in their current behavior during the project, and based on the post-implementation results, demonstrated a willingness to change behavior when provided with the appropriate support. The specific aims of the project were supported throughout the project. The primary aim was to investigate whether brief lifestyle modification counseling provided by a nurse practitioner (NP) could impact the self-management of diabetes among T2DM patients at one community mental health center (CMHC).

The Patient Activation Measures (PAM) assessments together represented the metric used to determine the efficacy of the program.
Thus, the project's purpose was to increase participants' engagement in their care to improve their medication and self-management skills, increase their physical activity, and to improve their dietary habits. The objective of this quality improvement project was to investigate whether brief lifestyle modification counseling could improve the level of patient self-management practiced by T2DM adult patients at the CMHC.

\section{Interpretation}

A lifestyle modification counseling program was included in the patient's current treatment at an outpatient CMHC and resulted in a positive relationship. This action demonstrated an increase in patient engagement. A large percentage of the patients in the clinic had diabetes and neglected to follow up with their PCP or refused to participate in their care; this project presented an opportunity for patients to obtain information from the CMHC concerning their diabetes management. Identifying patients with diabetes and depression is the first step to addressing health disparities arising from this condition as well as mental illness.

The project demonstrated the need for increased communication between the provider and the patient and for a proactive attitude toward diabetes self-management. New Beginnings Community Mental Health Center is one of Samaritan Daytop Village's outpatient mental health centers. Samaritan Daytop Village has been active in improving the quality of life for New Yorkers facing adversity for more than 50 years. Samaritan Daytop Village serves over 28,000 people annually at more than 50 facilities throughout New York City, Long Island, and upstate New York, and the organization continues to grow [28]. New Beginnings Community Mental Health Center will continue with the wellness program that will include diabetes groups and other patients' health concerns.

\section{Implications for Advanced Nursing Practice}

Psychiatric mental health nurse practitioners (PMHNP) provide mental health care to patients diagnosed with acute and chronic mental illness across the lifespan. The introduction into the mental health system can be a scary and confusing time for patients experiencing depression and diabetes. Improved communication between the healthcare provider and the patient are essential to greater outcomes and lowering overall expenditures in healthcare. The best time to educate patients is during the initiation of psychotropic medication for chronic diseases including diabetes. The patientprovider relationship may be strengthened by offering the patient a more personalized treatment experience which can assist the patient with incorporating the necessary lifestyle modifications for improved health. In service of this goal, the social cognitive theory can assist providers in grasping the personal and socio-structural components of treatment compliance [29].

\section{Future Research and Funding}

The combination of psychotropic medication, unhealthy lifestyles, and a decrease in primary care contributes to the development of uncontrolled diabetes. Future areas of research include identifying patients with dual diagnoses and implementing an educational program to heighten their understanding of diabetes to improve their overall health. In addition to education, another area of research could include the use of medical assistants to oversee primary care service referrals and to track primary care appointment attendance and medication compliance. 
Citation: Adepoju GA (2020) Evaluation of a T2DM Lifestyle Modification Counseling Program to Improve Patient Engagement in Diabetes Self-Care in a Community Mental Health Clinic. Int J Nurs Clin Pract 7: 319. doi: https://doi.org/10.15344/2394-4978/2020/319

Page 11 of 12

The agency provided no funding to support this quality improvement project, neither was outside funding received for this project. The project was completed using available resources within the agency setting. Funding may be necessary for the future to allow software and server upgrades to make provision for electronic intergrading of a diabetes monitoring tool.

The CMHC is a nonprofit agency providing care to the underprivileged population in an urban area: agencies are eligible for a greater range of grant funding than private agencies, and the monies could be used to include health promotion activities to support healthier lifestyles. Grant funding could assist with hiring additional staff such as health coaches [3]. This strategy has been identified as an evidence-based approach to improving patient health, especially among patients with diabetes. Such a program would present a unique solution to addressing health disparities in the community.

The findings from this quality improvement project indicate that patients increased adherence to self-manage their care with adequate support from the clinic. The sustainability of lifestyle modification counseling will increase as providers and therapists continue to add it to their mental health sessions. The results demonstrated that an increase in post-implementation lifestyle modification counseling helps patient engagement. The intervention was statistically significant and demonstrated that the introduction of a lifestyle modification counseling increased patient engagement. The results were disseminated to the clinic staff, providers, and executive staff members through a virtual teleconferencing platform.

Aguiar et al. [30] observed many components of lifestyle T2DM prevention interventions that include diet and aerobic and resistance training. This combined approach was effective in reducing weight and improving impaired fasting glucose status, glucose tolerance, dietary, and exercise outcomes in adult populations who were at risk for developing pre-diabetic symptoms [30]. Before the lifestyle modification counseling program was implemented, patients with T2DM who followed up or engaged in their care were identified in the PAM13 pretest median score (42.03). Post-implementation median scores indicating follow up or engagement in care increased to 45.86 This means the intervention had a significant impact in raising the PAM13 scores and supported the PICOT question.

The findings from this quality improvement project indicate that patients increased adherence to self-manage their care with adequate support from the clinic. The sustainability of lifestyle modification counseling will increase as providers and therapists continue to add it to their mental health sessions. The results demonstrated that an increase in post-implementation lifestyle modification counseling helps patient engagement. The intervention was statistically significant and demonstrated that the introduction of a lifestyle modification counseling increased patient engagement. The results were disseminated to the clinic staff, providers, and executive staff members through a virtual teleconferencing platform.

\section{Competing Interests}

The author declare that there is no competing interests regarding the publication of this article.

\section{References}

1. nternational Diabetes Federation (2018) Global burden of diabetes. In Diabetes atlas (5th ed.). Brussels, Belgium.

2. American Diabetes Association (ADA) (2016) Standards of medical care in diabetes. Diabetes Care 34: 1-112.
3. American Diabetes Association (ADA) (2018a) Classification and diagnosis of diabetes: Standards of medical care in diabetes. Diabetes Care 41: 13-27.

4. U.S. Department of Health \& Human Services, Centers for Disease Control and Prevention (CDC) (2015) Diabetes report card 2014.

5. Yoon U, Kwok KK, Magkidis A (2013) Efficacy of lifestyle interventions in reducing diabetes incidence in patients with impaired glucose tolerance: a systematic review of randomized controlled trials. Metabolism 62: 303-314.

6. American Diabetes Association (ADA) (2014) Standards of medical care in diabetes. Diabetes Care 37: 14-80.

7. Kikuchi $Y$, Iwase M, Fujii H, Ohkuma T, Kaizu S, et al. (2015) Association of severe hypoglycemia with depressive symptoms in patients with type 2 diabetes: The Fukuoka Diabetes Registry. BMJ Open Diabetes Research and Care 3: 1-8

8. Dalsgaard EM, Vestergaard M, Skriver MV, Helle TM, Lauritzen T, et al (2014) Psychological distress, cardiovascular complications, and mortality among people with screen-detected type 2 diabetes: Follow-up of the ADDITION-Denmark trial. Diabetologia 57: 710-717

9. American Diabetes Association (2018) Economic costs of diabetes in the U.S. in 2017. Diabetes Care 41: 917-928.

10. Yu M, Zhang X, Lu F, Fang L (2015) Depression and risk for diabetes: A metaanalysis. Can J Diabetes 39: 266-272.

11. Rubin RR, Wadden TA, Bahnson JL, Blackburn GL, Brancati FL, et al. (2014) Impact of intensive lifestyle intervention on depression and health-related quality of life in type 2 diabetes: The Look AHEAD Trial. Diabetes Care 37: 1544-1553.

12. DiClemente CC, Prochaska JO (1982) Self-change and therapy change of smoking behavior: A comparison of processes of change in cessation and maintenance. Addict Behav 7: 133-142.

13. Hill L, Turner LW, Hunt SB, Perko M (2008) Managing diabetes: Use of the transtheoretical model. Journal of the Arkansas Medical Society 43: 6-7.

14. Kaur J (2014) A comprehensive review on metabolic syndrome. Cardiol Res Pract 2014: 943162

15. Penninx BW, Milaneschi Y, Lamers F, Vogelzangs N (2013) Understanding the somatic consequence of depression: Biological mechanisms and the role of depression symptom profile. BMC Med 1: 1-14.

16. American Psychiatric Association (2013) Diagnosis and statistical manual of mental disorders. Arlington, VA: American Psychiatric Publishing.

17. Deuschle M (2013) Effects of antidepressants on glucose metabolism and diabetes mellitus type 2 in adults. Curr Opin Psychiatry 26: 60-65.

18. Park M, Katon WJ, Wolf FM (2013) Depression and risk of mortality in individuals with diabetes: A meta-analysis and systematic review. Gen Hosp Psychiatry 35: 217-225.

19. Vassar M, Holzmann M (2013) The retrospective chart review: Important methodological considerations. J Educ Eval Health Prof 10: 1-7.

20. Derrick B, White P (2017) Comparing two samples from an individual Likert question. International Journal of Mathematics and Statistics 18: 1-13.

21. Laerd Statistics (2013) Wilcoxon signed-rank test using SPSS.

22. Hibbard $\mathrm{HH}$, Greene J (2013) What the evidence shows about patient activation: Better health outcomes and care experiences: Fewer data on costs. Health Aff (Millwood) 32: 207-214.

23. Hibbard JH, Greene J (2013) What the evidence shows about patient activation: better health outcomes and care experiences; fewer data on costs. Health Aff (Millwood) 32: 207-214.

24. Rippa I, Linna M, Ronkko I (2014) The effect of a patient portal with electronic messaging on patient activation among chronically ill patients: Control before-and-after study. J Med Internet Res 16: 257.

25. Harvey L, Briggs Fowles J, Xi M, Terry P (2012) When activation changes, what else changes? The relationship between change in patient activation measure (PAM) and employees' health status and health behaviors. Patient Educ Couns 88: 338-343.

26. Kim MJ, Mallory C (2017) Statistics For Evidence-Based Practice In Nursing 2nd Edn, Jones \& Bartlett Learning, USA. 
Citation: Adepoju GA (2020) Evaluation of a T2DM Lifestyle Modification Counseling Program to Improve Patient Engagement in Diabetes Self-Care in a Community Mental Health Clinic. Int J Nurs Clin Pract 7: 319. doi: https://doi.org/10.15344/2394-4978/2020/319

Page 12 of 12

27. Melnyk BM, Finest-Overholt E (2015) Evidence-based practice in nursing: A guide to best practice. Philadelphia PA: Wolters Kluwer.

28. Samaritan Daytop Village.

29. Thomas JJ, Moring JC, Harvey T, Hobbs T, Lindt A, et al. (2016). Risk of type 2 diabetes: Health care provider perceptions of prevention adherence. Appl Nurs Res 32: 1-6.

30. Aguiar EJ, Morgan PJ, Collins CE, Plotnikoff RC, Callister R, et al. (2014) Efficacy of interventions that include diet, aerobic and resistance training components for type 2 diabetes prevention: A systematic review with metaanalysis. Int J Behav Nutr Phys Act 11: 1-10. 